\title{
On the dispensability of run-off elections
}

\author{
Salvatore Barbaro
}

Accepted: 14 December 2020 / Published online: 26 January 2021

(C) The Author(s) 2021

\section{Introduction}

The attempt to abolish the run-off elections in North Rhine-Westphalia failed in court. The amended election law that aimed to impose plurality voting did not meet the constitution's requirements, according to the ruling of the state court in 2019. The government justified the targeted abolishment in view of low voter turnouts. The (directly) elected incumbents may lack legitimacy if up to two-thirds of total voters abstain from casting a vote in run-offs, as it is often observed. Plurality voting, thus, yields more legitimacy as the turnout tends to be significantly higher in the first-round elections. The constitutional court rejected this view. It states that voter turnout is one indicator among others to judge whether a voting scheme satisfies the "principle of majority". This principle may also be violated if a candidate takes office with only about 20 to 30 per cent of votes. In this respect, the distance to the majority (obviously, the court defines the majority as a strict majority of votes) should be considered when assessing the appropriateness of a voting rule.

The court approved the parliament's competence to assess which voting rule is most appropriate to comply with the "principle of majority decision". Since both the plurality and the run-off election have flaws, it is up to the legislator to decide which shortcomings it considers to be more acceptable. In the present case, the legislator's considerations were not sufficient from the court's point of view. In particular, by substituting the run-off election with plurality voting, the legislator had not sufficiently taken into account the effect of what the court called a fragmentation on the ballots. The more candidates there are, the lower the percentage of votes that is sufficient to be elected. As such fragmentation has increasingly taken place in recent years, the balance between run-off and plurality voting is different today than

Prof. Dr. S. Barbaro $(\bowtie)$

Department of Law and Economics, Johannes-Gutenberg University Mainz, 55099 Mainz, Germany

E-Mail: sbarbaro@uni-mainz.de 


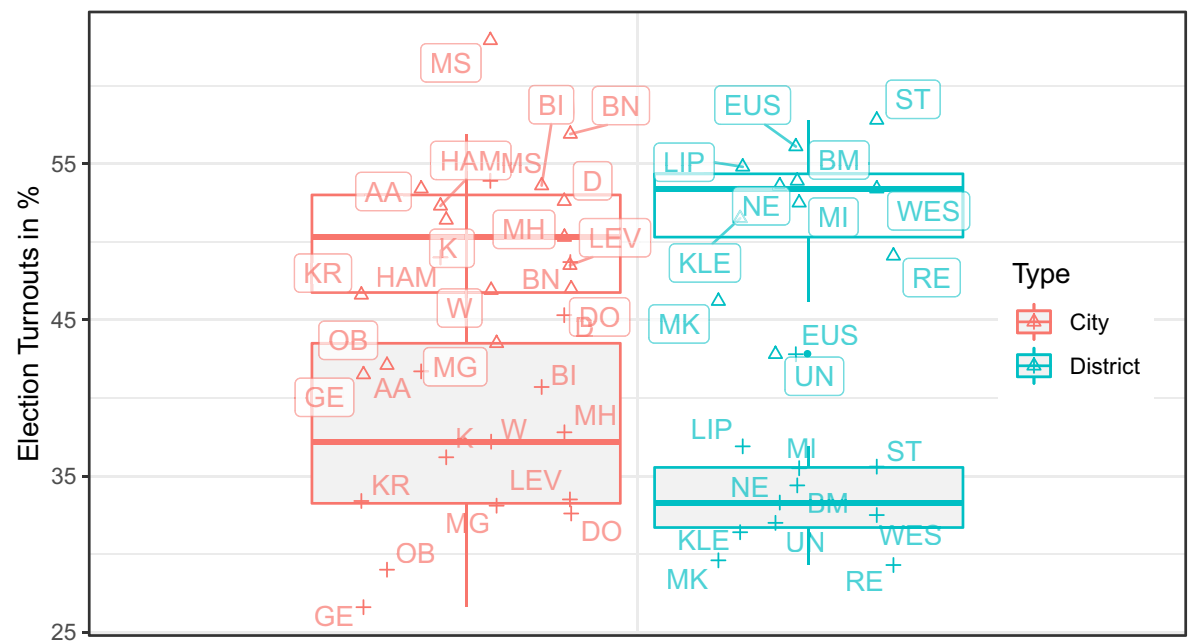

Fig. 1 Election turnouts. Local election in North Rhine-Westphalia 2020. Data source: Ministry of interior affairs

it was several years ago. This part of the court's statement refers to a law from 2007 with identical content. From 2007 to 2011, local incumbents were elected by plurality voting. The election law had already been subject to legal review, but the court confirmed the law at that time. While the legislative had sufficiently justified the former election-law reform in light of diminishing turnouts, this argument alone no longer complies with today's legal requirements.

The 2020 local elections, hence, took place under strict majority cum run-off. Twenty-six lord mayors and district chief executives (DCE) ${ }^{1}$ were elected by runoff after no candidate in the respective districts had cleared the 50\%-hurdle. The election turnouts remained on a low level, in particular in the (second-round) runoff elections. Figure 1 depicts the electoral participation. The unfilled box plots depict the election turnouts in the first-round elections, the grey-filled box plots the respective distribution in the run-offs. The framed labels indicate the districts and cities at the first-round elections, the unframed labels the respective values at the run-offs.

The average turnout in first-round elections was higher in districts than in cities. When run-off elections were held, the turnouts dropped in both cities and districts. Interestingly, the drop-down was more remarkable in the districts. On average, roughly half of the voters $(51.9 \%)$ went to the ballot box when they were called to vote for the first time. For the run-off, only $36.6 \%$ of all voters wished to express their opinion in the polling station. In districts, roughly a third of voters participated in the second round.

In this respect, the need to reconsider run-off elections is still given and considerations on how to improve the present voting systems for local incumbents should continue. In this paper, we argue in favour of simple-majority voting for several

\footnotetext{
1 In German: Oberbürgermeister/innen and Landrät/innen
} 
reasons. In particular, we argue that simple-majority voting is not only superior to other voting schemes but makes second-round run-offs dispensable. Thus, the runoff elections should not be substituted by first-past-the-box, but rather by a superior voting scheme.

The paper is organized as follows: Section 2 summarizes some axiomatics of voting. We present some results of the axiomatic voting theory, which are commonly used in the social-choice theory in Subsection 2.1. We argue in favour of the Condorcet principle (first axiom) and impose the requirement of an unrestricted domain as the second axiom. Based on these considerations, we demonstrate in Subsection 2.2 that neither strict-majority voting nor plurality rule can satisfy the justmentioned axioms simultaneously. Further, we will demonstrate that even positional rules violate basic conditions of voting (Subsection 2.3) and we will discuss several aspects of simple-majority voting in Subsection 2.4.

In Section 3, then, we will demonstrate that run-off election are dispensable under the superior voting scheme of simple majority. Due to Arrow's trail-blazing impossibility theorem ${ }^{2}$, every voting rule must have flaws. Simple-majority rule is no exemption. Therefore, we will deal with indeterminacy as the bugaboo of the simple-majority rule in Section 4. We will show that even if there is no Condorcet winner, the simple-majority rule might be decisive through an instant run-off for a wide range of preference profiles. Section 5 summarizes the results and provides some concluding remarks.

\section{Social choice considerations on voting}

\subsection{Axiomatics}

What kinds of systems, whether for electing government leaders or mayors, can best reflect the voters' wishes? To provide an answer to this question, one first needs to state a general framework to assess an electoral outcome. Most political scientists and social choice theorists would agree that any good electoral method ought to satisfy several axioms. In his path-breaking work, Arrow (1963) imposes four conditions of reasonable sensitivity, which should be simultaneously satisfied by a collective decision. Any social ordering violates at least one of these "rational and democratic" (as Arrow called these conditions, "with some plausibility" Sen (2009, p. 93)) requirements if there are more than two alternatives. As this outstanding result applies to any social ordering, it must also hold for any electoral system. Among these requirements is one about independence from irrelevant alternatives, I. It requires that the voters' choice between candidates $x$ and $y$ should not depend on their views regarding some third candidate $z$. In particular, if a majority of voters prefer $x$ to $y$, the election scheme should not allow $y$ to take office by adding a third candidate $z$. Today's voting theory uses some other axioms, which are closely related to those imposed by Arrow. Most represent and imply subsets of the former. For a good overview, see Sen $(2017$, Ch. 5*).

\footnotetext{
2 There exists a large number of proof. Among them is the often-used proof by Sen (1995b).
} 
We return to the court's decision on the new electoral law and the requirements to legislative considerations imposed. The principle of the majority decision shall be the basis for assessing a voting rule. It is helpful to formally define the principle of majority decision. Before we pursue, we are going to introduce some notions.

We denote individual preferences of $n$ voters with $\left(\succ_{1}, \ldots, \succ_{n}\right)$. We use the common notation $\succ_{i}$ for the anti-symmetric (strict) individual preference and with $N(a \succ b)$ the number of voters who strictly prefer $a$ to $b$. Accordingly, $\gtrsim_{i}$ denotes the weak preference binary relation ("is at least as good as") ${ }^{3}$. Further, we denote a strict social preference of $a$ over $b$ with $a P b$ (and a weak one by $a R b$ ).

Equipped with these notations, we can define the principle of majority decision (PMD) in line with Sen (2017, Ch. 5*) as follows:

Definition 1 (Principle of majority decision, PMD) The principle of majority decision holds if and only if $\forall x, y \in \mathfrak{B}: x P y \Leftrightarrow[N(x>y)>N(y>x)]$.

If there are two candidates on the ballot $\mathfrak{B}=\{x, y\}$, then candidate $x$ should be the election winner if and only if more voters prefer him or her over $y$ than vice versa. In that case, $y$ should be ranked socially second. This point of view can easily be regarded as generally accepted. At least, we do not know of any literature source that contradicts this view.

Now, by combining the PMD with the axiom I, it can be said that a third candidate may be ranked socially first, second, or third. In any case, his or her candidacy should not lead to a violation of PMD. The defeated candidate $y$ should not be able to overtake $x$. In particular, the voting rule should not give $y$ the opportunity to become the election winner simply by adding a third candidate to the ballot. Otherwise, a candidate may be elected to office even though a majority regards an opposing candidate as superior.

As a consequence, a candidate that is never defeated, hence wins all head-to-head comparisons, should be elected. Such a candidate is called the Condorcet winner.

Definition 2 (Condorcet winner) Let $\mathfrak{B}$ be the set of candidates and $\mathcal{N}$ the set of voters. A candidate $x \in \mathbf{B}$ is a Condorcet winner if and only if $\forall y \in \mathfrak{B}, y \neq x$ : $N(x>y)>N(y>x)$. We denote the case that $x$ is a Condorcet winner by $\exists \mathcal{C}(x)$.

Accordingly, we can define a Condorcet loser. It is the candidate beaten in every head-to-head match-up.

For our purposes, it is sufficient to impose two axioms that a voting rule should achieve simultaneously. The first follows from the considerations just described.

Axiom 1 (Majority consistency, C) A voting rule should be such that if there is a Condorcet winner, i.e., $\exists \mathcal{C}(x), x$ is elected.

The second axiom that shall be satisfied is Arrow's ${ }^{4}$ axiom $\mathbf{U}$.

\footnotetext{
3 Almost all notations used in this paper are taken from Sen (2017) as a standard reference in social choice. There are two exemptions: the negation is denoted by $\neg$ instead of $\sim$ and the logical 'or' is represented by $\underline{V}$. Further notations are defined in this paper.

4 Arrow (1963, p. 24f) calls this requirement collective rationality. It includes a stipulation that preferences be logically well behaved, i.e., transitive and complete.
} 
Table 1 A Condorcet winner without any "vote" and a Condorcet loser as plurality winner

\begin{tabular}{llll}
\hline voter group & $\mathcal{A}$ & $\mathcal{B}$ & $\mathcal{C}$ \\
voter shares & $47 \%$ & $49 \%$ & $4 \%$ \\
\hline Rank 1 & y & z & w \\
Rank 2 & x & x & x \\
Rank 3 & w & y & y \\
Rank 4 & z & w & z \\
\hline
\end{tabular}

Axiom 2 (Unrestricted domain, U) The domain of any voting rule must include all logically possible combinations of individual orderings.

Arrow (1963, p. 24f) describes the axiom's importance by stating that $\mathbf{U}$ "is a restriction on the form of the social welfare function, since, by definition of an admissible set of individual orderings, we are requiring that, for some sufficiently wide range of sets of individual orderings, the social welfare function give rise to be a true social ordering".

\subsection{Irrelevance of votes}

It is straightforward to demonstrate that neither strict majority rule nor first-pastthe-box can satisfy the two axioms simultaneously. Due to $\mathbf{U}$, a single example is sufficient. Consider a ballot $\mathfrak{B}$ consisting of four alternatives, $\mathfrak{B}=\{x, y, z, w\}$. The voters can be divided into three groups with identical preferences within each group. Let the individual orderings be as presented in Table 1.

As can easily be checked, $x$ is the Condorcet winner. In head-to-head comparisons, $x$ beats all other candidates on the ballot with $53 \%(y), 51 \%(z)$, and $96 \%(w)$. The candidate $z$, however, is the winner under plurality voting even though he or she is defeated in a head-to-head comparison by $y$ (who is the winner under strictmajority voting cum run-off election ${ }^{5}$ ), and also by $x$. Thus, the plurality-winner is the candidate who is socially ranked third by counting the head-to-head victories. Note further, that the Condorcet winner $x$ fails to enter into the run-off under strictmajority voting.

Let us define a "vote" as the voter's first preference and let us assume menuindependence 6 . This means that under any non-positional electoral system, a voter casts a vote for his or her favourite candidate. As can be seen by the example in Table 1, a Condorcet winner might be a candidate without any vote.

For reasons of clarity, we impose the following lemma.

Lemma 1 (Irrelevance of votes) For assessing voters' wishes, votes are irrelevant for a wide domain of preference profiles.

\footnotetext{
5 By assuming that the same voters participate at both election rounds, thus neglecting the observable turnout-drop.

6 To keep things simple, we in fact assume menu-independent preference orderings (Sen 1995a). This assumption is not self-evident. If the voter were offered a choice over a subset of the set over which that voter has expressed a ranking, we may or may not presume that the voter would stick to the same overall ranking applied to this particular subset. There are, however, reasonable arguments for scrutinize menudependency see Sen (1993).
} 
Table 2 Borda count: preference profile with four candidates

\begin{tabular}{llll}
\hline voters & 47 & 49 & 4 \\
\hline Rank 1 & x & y & w \\
Rank 2 & y & x & x \\
Rank 3 & w & w & y \\
\hline
\end{tabular}

Lemma 1 is crucial for dealing with ruling of the court. As mentioned above, the court requires the legislative to balance the low turnout with the number of votes a candidate needs to be elected to office. Our analysis shows that the court focuses on a mostly irrelevant variable. The number of votes is an appropriate indicator only if a candidate receives more than half of the votes total. In this case, the candidate is the winner under any democratic voting system. This is due to $\exists \mathcal{S}(x) \rightarrow \exists \mathcal{C}(x)$, which does not hold vice versa. In this connection, $\exists \mathcal{S}(x)$ denotes that $x$ is winner under strict-majority voting. The discrepancy between the number of votes and the "majority" was also recently emphasized by Sen (2017, Ch. A4*).

Lemma 1 further implies that neither strict-majority rule nor plurality voting satisfy the axioms $\mathbf{C}$ and $\mathbf{U}$ simultaneously. The Condorcet winner may fail to enter the run-off under the strict-majority rule. A plurality winner may well be defeated in a head-to-head majority vote by several other candidates. Moreover, even a Condorcet loser, the candidate who is defeated by all other candidates, might be elected under first-past-the-box. Table 1 also illustrates this insight. The plurality winner $z$ is also a Condorcet loser. For a general proof, see Saari and Newenhizen (1988).

The crux of the matter is that under almost all voting rules, a voter is allowed only to unveil his or her favourite candidate rather than ranking them all. If just two candidates are on the ballot, this restriction makes no difference. But with more than two candidates, it can matter a great deal. Candidate $z$ 's landslide under firstpast-the-box and $y$ 's electoral success under strict-majority voting cum run-off are due to voter preferences being re-evaluated. As voters' ranking (ordering) cannot be disclosed on the ballot, both voting schemes can be interpreted as a voting rule in which all but the first-placed candidate are tied for second place. Thus, considering Table 1, instead of $y \succ_{\mathcal{A}} x \succ_{\mathcal{A}} w \succ_{\mathcal{A}} z$, the preferences of the voters in this group are re-judged as $y \succ_{\mathcal{A}} x \sim_{\mathcal{A}} w \sim_{\mathcal{A}} z$. The preferences of the other voters are re-judged similarly. This re-judgement leads to a violation of the positive responsivenessproperty $(\mathbf{S})^{7}$. By violating $\mathbf{S}$, the Condorcet winner can even be beaten by his or her rivals. A more general examination on this topic is provided by Barbaro (2020).

\subsection{Positional voting rules}

It immediately follows from the considerations in the last subsection that any voting rule that satisfies axioms $\mathbf{C}$ and $\mathbf{U}$ is one which allows voters to unveil their full

\footnotetext{
${ }^{7}$ Positive responsiveness requires that the relationship between individual and social preferences must be positive, i.e., if $x$ is considered to be socially as good as $y$ in some situations and $x$ rises in someone's preference vis-a-vis $y$ and does not fall in anyone's preference, then $x$ must now be regarded as socially strictly better than $y$.
} 
Table 3 Borda count: preference profile with four candidates

\begin{tabular}{llll}
\hline voter shares & 47 & 49 & 4 \\
\hline Rank 1 & x & y & w \\
Rank 2 & y & z & x \\
Rank 3 & z & x & y \\
Rank 4 & w & w & z \\
\hline
\end{tabular}

preference orderings. However, it is a well-elaborated result that some positional rules (voting schemes which consider individual preference orderings) violate the axiom of neutrality and thus $\mathbf{I}$. The most common-known positional rule is the Bordacount, which was often used in pubs in the days of Arrow and is in use to determine the winner in a European-wide singling contest. The following example illustrates the flaw of Borda count; the expert reader may skip the rest of this subsection.

Consider the following preference profile of 100 voters, depicted in Table 2.

The Borda count yields $x$ as election winner (a nice result, as $x$ is also the Condorcet winner).

$$
\begin{array}{ll}
x: & 1 \times 47+2 \times 49+2 \times 4=153 \\
y: & 2 \times 47+1 \times 49+3 \times 4=155 \\
w: & 3 \times 47+3 \times 49+1 \times 4=292
\end{array}
$$

Now consider that the defeated candidate $y$ proposes the candidacy of a fourth candidate, $z$. The extended profile is given in Table 3 .

By adding a fourth candidate, we find:

$$
\begin{array}{ll}
x: & 1 \times 47+3 \times 49+2 \times 4=202 \\
y: & 2 \times 47+1 \times 49+3 \times 4=155 \\
z: & 3 \times 47+2 \times 49+4 \times 4=255 \\
w: & 4 \times 47+4 \times 49+1 \times 4=388 .
\end{array}
$$

Thus, $y$ achieves a landslide victory.

It is well-elaborated that similar effects can also be found in other positionalvoting rules, for an overview cf. Saari (2000).

\subsection{Simple-majority voting, SMR}

The most frequently proposed method by social-choice theorists is the simple-majority rule, SMR (see, for instance, Dasgupta and Maskin (2004); Maskin and Sen (2016)). SMR satisfies the two axioms simultaneously for two reasons. First, under SMR, the voters are allowed to unveil their preference orderings. Second, the no rejudgement of preferences takes place, and third, SMR satisfies the conditions I and $\mathbf{S}$ (among others). This finding follows from May's theorem. Consider the following properties:

1. Neutrality $(\mathbf{N})$ requires that the rule of choice should not discriminate between alternatives, and whichever criterion permits us to say that $x$ is socially as good as $y$ should also sufficiently declare $w$ to be socially as good as $z$, after replacing $x, y$ by $w, z$. Note that $\mathbf{N} \rightarrow \mathbf{I}$, as mentioned above. 
2. Anonymity (A) holds that who you are should not determine your influence on the election. All individuals should count the same and the name-tags, which label the preference set of an individual, should not matter. If anonymity is postulated, there cannot be a dictator in the Arrovian sense, cf. Gaertner (2006, p. 33), and

\section{Positive responsiveness $(\mathbf{S})$.}

A doctorate version of May's Theorem (see Sen $\left(2017\right.$, Ch. $\left.5^{*}\right)$ ) is

Theorem 1 (May's Theorem) Conditions $\mathbf{U}, \mathbf{A}, \mathbf{N}$, and $\mathbf{S}$ are together necessary and sufficient for a decisive collective choice rule (CCR) to be the method of majority decision.

Despite the fact that the SMR is widely regarded as the superior voting method (we will present the so-called Maskin's theorem in Section 5), it is seldom (if at all) in use. There may be three main reasons for this. Firstly, SMR lacks determinacy; i.e., it is not decisive. There may be no Condorcet-winner at all, but a cycling pattern instead. In this case, a decisive rule for an instant run-off is required. We will shed light on this case in the next Section. Secondly, SMR is regarded as too complicated and is likely to confuse voters. Thirdly, tallying is too complex.

Concerning the last-mentioned objection, tallying has become much easier through digitalization ${ }^{8}$. Moreover, the voting system used in most German states for local elections, which allows for cumulation and vote-splitting, appears to be at least as complicated to tally as simple-majority voting.

Regarding the second-mentioned objection, that SMR is too complicated, some remarks should be given. In fact, two German ministers of interior affairs expressed such a fear to the author after having been confronted with the proposal in favour of SMR for directly-elected incumbents. We assume that the fear is due to a misinterpretation regarding the SMR. The voters are allowed to unveil their orderings, they are not forced at all.

Consider a politically-interested citizen, $i$, who has a preference ordering over, say, six candidates on a ballot. If now this citizen wishes to place the ranking numbers one to six on the ballot, then there can be no complexity from the voter's point of view.

What sitting incumbents seem to have in mind is a voter, $j$, who knows which of the candidates he or she ranks first, but is indifferent regarding all the other candidates. In this case, the voter can place a cross or the number 1 in the line of the ballot paper where his or her favourite candidate is listed. The other candidates, however, receive no ranking-number by the voter. Thus, for voter $j$, nothing changes by replacing strict-majority voting or plurality rule by SMR. Voter $i$, on the other hand, is better-off, since the voter can now specify his or her preference ordering and it is not re-judged as with non-positional voting systems.

To illustrate this, denote the six candidates on the ballot $\mathfrak{B}$ by $\{A, \ldots, F\}$. Further, let candidate $C \in \mathfrak{B}$ be the candidate favoured by voter's $j$. Then $j$ 's preferences are

\footnotetext{
8 A new package for the statistical software $\mathbf{R}$, labelled "vote", is freely available and allows to tally quickly under different voting rules. Hana Ševčíková and the author have recently added the simple-majority rule with run-off options.
} 
given by $C \succ_{j} A \sim_{j} B \sim_{j} D \sim_{j} E \sim_{j} F$ and accordingly considered when it comes to tallying his ballot.

One may also consider a third voter, who has a favoured candidate. He or she regards four out the remaining five candidates as indifferent. Nevertheless, this voter regards one candidate as the worst compared to all other candidates. In this case, this voter places a ranking-number " 1 " to his or her favoured candidate and a " 6 " to the candidate he or she regards the worst. When the voter's ballot is tallied, the worst candidate is defeated by all other candidates. In this respect, it is worth considering that while voters have no strict (anti-symmetric) preferences over a number of tuples they still regard a candidate as the worst choice. SMR provides these voters with the opportunity to express their opinion. Recall the last Bundestag election. Two current MP from a far-right party won the election for the directly-elected MP. They were elected under plurality rule and received roughly one third of the total votes. Even if it is speculative, let two-third of the respective voters in the two electoral districts regard them as the worst options. In this case, the opinion of a two-third majority played no role and the opinion of the minority was decisive. Under SMR, however, it would not be possible for a Condorcet loser to take a seat in the parliament as a directly-elected MP. In this context, see the elaborations over the myth of Hitler as a majority winner by Sen (2017, Ch. A4).

Summing up, as no voter is forced to disclose any preference ordering, no voter can be worse-off when applying SMR. On the other hand, all voters who have more than only one favoured candidate and regard all other alternatives as indifferent, are better-off. At no point, we find an example where a voter is faced with a decision he or she cannot make due to complexity. Not SMR is tricky, but the voters' judgements on alternatives might be complicated.

Finally, we can not brush aside the objective that SMR is not decisive. We will, therefore deal with the phenomenon of indeterminacy in Section 4.

\section{Dispensability of second-round run-off elections}

The starting point for our analysis was the often observed low turnouts in run-off elections. It repeatability led to raising the question on the legitimacy and to attempts to abolish them by substituting strict-majority voting by plurality rule.

In this Section, we will demonstrate that a second-round run-off is dispensable under SMR. First of all, a run-off is only required in cases where there is no Condorcet winner on a ballot. Otherwise, he or she is elected in the first round. Thus, we are only dealing with those situations where there is no Condorcet winner.

Firstly, it follows from Lemma 1 that a run-off should be held between the two candidates with the most and second-most pair-wise victories (cf. Sen 2017, Ch. A4*). This rule ensures that a Condorcet loser can not become an election winner. Secondly, a run-off is an election between two candidates (tuple). The voters are asked to unveil their preferences over this tuple. Under SMR, voters have already expressed their preferences on all conceivable tuples in the first ballot. Thus, voters do not need to be called to the polling station for a second time, as all necessary information is already available. 
Thus, the need for a (second-round) run-off under strict-majority rule arises solely from the fact that voters are not allowed to reveal their entire preference orderings when filling out the ballot. If they could, it would become clear that a majority would prefer, say, $x$ over $y$ and hence, $x$ would emerge as the winner of an instant run-off. In practice, we call voters to an election and allow them to disclose only a subset of their preferences. In a second-round run-off then, voters are allowed to express additional information from their preferences. This is neither an efficient deployment of public resources nor the time budget of the citizens.

\section{What, if there is no Condorcet winner?}

For the following discussion, it is helpful to consider three different cases.

1. There is one (and only one) candidate with the most and one (and only one) candidate with the second-most head-to-head victories. These candidates compete against each other in an instant run-off. The cast ballots provide the voter preferences over this tuple. Thus, the election winner is the candidate who is supported by a majority over his or her run-off opponent. This case also applies to the situation where two candidates achieve the most victories. The run-off then takes place between these two candidates.

2. There is more than one candidate with the most victories, or there is more than one candidate with the second-most victories. In this case, we have a run-off with more than two candidates on the ballot $\mathfrak{B}_{\text {ro }}$. Then, either there is a Condorcet winner on the ballot $\mathfrak{B}_{\text {ro }}$, or we have a second run-off, also instant. This procedure will be repeated on a smaller ballot $\mathfrak{B}_{\text {ro }}^{(2)} \subset \mathfrak{B}_{\text {ro }}$ until a Condorcet winner is determined or a full-majority cycle (see next item).

3. As in the second case, the run-off takes place between more than two candidates and these candidates constitute a full-majority cycle (see Sen 2017, p. 416). In the case, where no candidate in $\mathfrak{B}_{\text {ro }}$ or any subsequent run-off wins more often the head-to-head match-ups than any other, we have an inescapable intransitivity.

The debate on what has to happen in such cases is still ongoing in literature and has become a pretty key issue. There is no necessarily appropriate answer. Any possible rule must violate at least one of Arrow's conditions. In such cases (and only in such cases of an inescapable intransitivity), the instant run-off takes place under a voting rule other than SMR. There are several ways this can be done. It seems reasonable to rely on rank-order rules 9 . In our estimation, the Borda count is an appropriate voting system for determining the election winner when the preference profile is intransitive, even though the Borda-count procedure violates the principle of neutrality. The reason for the appropriateness-claim is that it is not possible to add an additional candidate to either the closed set $\mathfrak{B}_{\text {ro }}$ or to a strict subset of this. Unlike a first-round election, where the number of candidates is

\footnotetext{
9 Dasgupta and Maskin (2004) propose the following in case of a full-majority cycle: If no one obtains a majority against all opponents, then among those candidates who defeat the most opponents in head-tohead comparisons, select as the winner the one with the highest rank-order score.
} 
not limited (we neglect the rules for the minimum voting age, etc.), in a run-off election the number of candidates is fixed and a candidate $y$ beaten by $x$ cannot overtake $x$ by adding additional candidates.

These examinations should be suitable to reconsider the relevance of indeterminacy.In this paper's introductory chapter we had somewhat casually titled it as a bugaboo of simple-majority voting. Even if there is no Condorcet winner in the first round, there may be one in an instant run-off. Since the run-off elections can (instantaneously) be repeated $n$ times (by reducing the ballot each time), the voting rule is only then not decisive where there is no Condorcet winner even on ballot $\mathfrak{B}_{\text {ro }}^{n}$. This should give reason to consider the simple-majority rule as appropriate voting systems whenever an office is to be filled.

\section{Conclusion}

Second-round run-off elections can be scrutinized for several good reasons. All the same, we do not see reasonable arguments for substituting strict-majority voting cum run-off by plurality rule. Based on the principle of majority decision, the election should be won by the Condorcet winner (if there is one). Under unrestricted domain (U),both strict-majority voting and plurality rule show considerable flaws. Instead of bringing forth the Condorcet winner as the winner, even the Condorcet loser might take office under first-past-the-box. Under both rules, it is possible that the Condorcet winner is ruled out.

Given the existence of a Condorcet winner, the only voting method that fulfills the requirements of the PMD, is the simple-majority voting (SMR). Nevertheless, the fundamental insight of Arrow's impossibility theorem remains, according to which every possible voting rule must have some flaw. Sometimes, even the simplemajority rule still fails to work well due to its indeterminacy if preference profiles lead to a full-majority cycle. In such a case, an instant run-off might bring forth an election winner. We argue that the run-off should be between the candidates with the most and second-most pair-wise victories. If the run-off ballot constitutes an inescapable intransitivity, then the run-off has to be modified in order to identify a winner. Let us assume that in such a case the winner would be determined by the plurality rule (which we do not recommend). Even in this case, the serious flaws of the plurality rule would only come to bear in the particular case of an inescapable intransitivity. Thus, only in this particular case, the election outcome may be as faulty as under plurality rule. In any other case, the SMR is superior. This result has been generalized by Maskin (1995).

Theorem 1 (Theorem of Maskin) Let $F$ be a $n$-person (n odd) collective-choice rule (CCR) satisfying $\mathbf{A}, \mathbf{N}, \mathbf{P}$ and $\mathbf{I}$. Suppose that $\mathfrak{R}$ is a domain of anti-symmetric preferences (i.e., strict preferences) on which $F$ is a social-welfare function (thus, transitive). Then SMR is also a social-welfare function on $\mathfrak{R}$. Moreover, unless $F$ is itself SMR, there exists some domain $\mathfrak{R}^{\prime}$ on which SMR is a social-welfare function, but $F$ is not. 
In this contribution, Maskin showed the robustness and in this sense the superiority of SMR. Any alternative to SMR, and non-positional rules in particular, are inferior in the fulfillment of the just-mentioned conditions.

Taking into account the results from the social-choice literature, there is no reasonable argument in favour of maintaining traditional voting rules. Instead, if second-round run-off elections are to be avoided, the current voting system should be replaced by a superior one.

We discussed some concerns that exist in connection with SMR, in particular the alleged complexity. We see no compelling reasons against the introduction of SMR. Neither for the election of the state's president nor when the mayor's seat is to be filled.

Acknowledgements For helpful comments and fruitful discussion I am indebted to Marlene Wienold, Susanne Fischer, and Marcel Hürter. Hana Ševčíková, one of the maintainer of the R-package "vote", provides some useful extensions in order to simulate election results by considering the run-off approach, which has been discussed in this paper.

Funding Open Access funding enabled and organized by Projekt DEAL.

Open Access This article is licensed under a Creative Commons Attribution 4.0 International License, which permits use, sharing, adaptation, distribution and reproduction in any medium or format, as long as you give appropriate credit to the original author(s) and the source, provide a link to the Creative Commons licence, and indicate if changes were made. The images or other third party material in this article are included in the article's Creative Commons licence, unless indicated otherwise in a credit line to the material. If material is not included in the article's Creative Commons licence and your intended use is not permitted by statutory regulation or exceeds the permitted use, you will need to obtain permission directly from the copyright holder. To view a copy of this licence, visit http://creativecommons.org/licenses/by/4. $0 /$.

\section{References}

Arrow, K.J. 1963. Social choice and individual values. New York: Wiley.

Barbaro, S. 2020. Elections in a deliberative democracy: on the inappropriateness of a class of voting rules to identify a Condorcet winner, Discussion Paper. GSME-Discussion paper, University of Mainz.

Dasgupta, P., and E. Maskin. 2004. The fairest vote of all. Scientific American 290:92-97. https://doi.org/ 10.1038/scientificamerican0304-92.

Gaertner, W. 2006. A primer in social choice theory. LSE perspectives in economic analysis. New York: Oxford University Press.

Maskin, E. 1995. Majority rule, social welfare functions, and game form. In Choice, welfare and development, ed. K. Basu, P.K. Pattanaik, and K. Suzumura, 100-109. Oxford: Clarandon.

Maskin, E., and A. Sen. 2016. How majority rule might have stopped Donald Trump. The New York Times, April 28. https:/www.nytimes.com/2016/05/01/opinion/sunday/how-majority-rule-mighthave-stopped-donald-trump.html. Accessed 10 December 2020.

May, K.O. 1952. A set of independent necessary and sufficient conditions for simple majority decision. Econometrica 20:680-684.

Saari, D.G. 2000. Mathematical structure of voting paradoxes: Ii. positional voting. Economic Theory 15:55-102.

Saari, D.G., and J.V. Newenhizen. 1988. The problem of indeterminacy in approval, multiple, and truncated voting systems. Public Choice 59:101-120. https://doi.org/10.1007/bf00054447.

Sen, A. 1993. Internal consistency of choice. Econometrica 61:495-521. https://doi.org/10.2307/2951715.

Sen, A. 1995a. How to judge voting schemes. Journal of Economic Perspectives 9:91-98. https://doi.org/ 10.1257/jep.9.1.91.

Sen, A. 1995b. Rationality and social choice. American Economic Review 85(1):1-24.

Sen, A. 2009. The idea of justice. London: Allen Lane.

Sen, A. 2017. Collective choice and social welfare. Cambridge: Harvard University Press. 